\title{
Dynamics of Wicking in Silicon Nanopillars Fabricated with Interference Lithography and Metal-Assisted Chemical Etching
}

\author{
Trong Thi Mai, ${ }^{\dagger}$ Chang Quan Lai, ${ }^{\dagger}$ H. Zheng, ${ }^{\dagger}$ Karthik Balasubramanian, ${ }^{\S}$ K. C. Leong, ${ }^{\prime \prime}$ P. S. Lee, ${ }^{\S}$
} Chengkuo Lee, ${ }^{\dagger}$ and W. K. Choi $*^{* \dagger, \dagger, \perp}$

${ }^{\dagger}$ Department of Electrical and Computer Engineering, National University of Singapore, Singapore 117576

${ }^{\ddagger}$ Advanced Materials for Micro- and Nano-Systems Programme, Singapore-MIT Alliance, Singapore 117576

${ }^{\S}$ Department of Mechanical Engineering, National University of Singapore, Singapore 117576

"GLOBALFOUNDRIES Singapore Pte. Ltd, Singapore 738406

${ }^{\perp}$ NUS Graduate School for Integrative Sciences and Engineering, National University of Singapore, Singapore 117456

Supporting Information

ABSTRACT: The capillary rise of liquid on a surface, or "wicking", has potential applications in biological and industrial processes such as drug delivery, oil recovery, and integrated circuit chip cooling. This paper presents a theoretical study on the dynamics of wicking on silicon nanopillars based on a balance between the driving capillary forces and viscous dissipation forces. Our model predicts that the invasion of the liquid front follows a diffusion process and strongly depends on the structural geometry. The model is validated against experimental observations of wicking in silicon nanopillars with different heights synthesized by interference lithography and metalassisted chemical etching techniques. Excellent agreement between theoretical and experimental results, from both our samples and data published in the literature, was achieved.
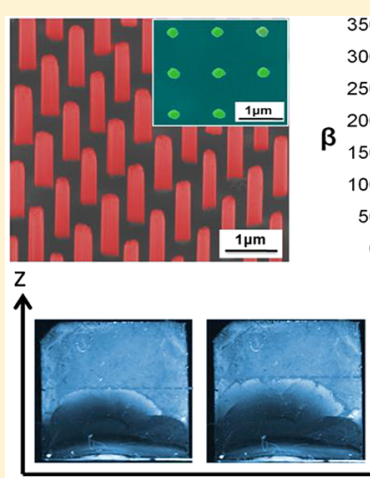

\section{INTRODUCTION}

The wicking of fluids on micro/nano-textured surfaces is a subject that has received much attention lately because of its many engineering applications, which include thermal management for microchips, ${ }^{1-4}$ biomedical devices, ${ }^{5-9}$ sensors ${ }^{10,11}$ and industrial processes such as oil recovery. ${ }^{12}$ Wicking is defined as the imbibition of a wetting liquid into the space between micro/nano- structures by means of a thin film that extends from a reservoir of the wetting liquid (e.g., a droplet, beaker of liquid etc.). Although wicking has been shown to take place on both regular ${ }^{13-16}$ and irregular patterns of structures, ${ }^{17-19}$ quantitative models have only been proposed on ordered array surfaces, with square arrays of micropillars being the most studied system due to the ease of fabrication and analysis.

There are a few publications on the conditions and effects of structural geometry on the wicking phenomenon. For instance, Martine et al. ${ }^{15}$ studied the advancing contact angles of fluid (60\% NEB31A3/40\% EC solvent) on silicon nanopillars based on the theory of wicking. On the other hand, Extrand et al. ${ }^{16}$ concentrated on the prediction of the maximum radius/ distance an oil droplet can spread on a superoleophilic surface consisting of regular square pillars. However, none of these works examined the dynamics or velocity of wicking.

Washburn ${ }^{20}$ predicted the wicking dynamics of capillary flow in cylindrical tubes to follow a diffusive process such that the impregnated length is proportional to the square root of time.
Bico et al. ${ }^{13}$ examined wicking on silicon micropillars and characterized the dynamics of invasion by balancing the capillary and viscous forces. Bico et al. ${ }^{13}$ first treated the viscous force on the wicking film as that sustained by a flow on a flat plane without any structures, and then, to take into account the effect of the micropillars, the viscous force was enhanced by an empirical value $\beta$. More recently, Hay et al. ${ }^{21}$ attempted to explain the dynamics of wicking using a hydraulic diameter approximation. However, in this model, a fitting parameter, i.e., the tapered angle of the pillars, was introduced in order to fit Bico's data. Apart from those studies, Ishino et al. ${ }^{14}$ divided the wicking of liquid on silicon micropillars into 2 cases, namely, the short pillar (the height of the pillars is smaller than the period of separation) and long pillar (pillar height is larger than the period) regimes. For the short pillar regime, the effect of viscous dissipation on the pillar walls is ignored. For the latter, the viscous force is derived using an approximation that the cutoff distance (the distance beyond which the effect of the pillars is negligible) is equal to the period. Although the resulting equations fitted the experimental data in the second regime, the results in the first regime

Received: June 4, 2012

Revised: July 11, 2012

Published: July 11, 2012 


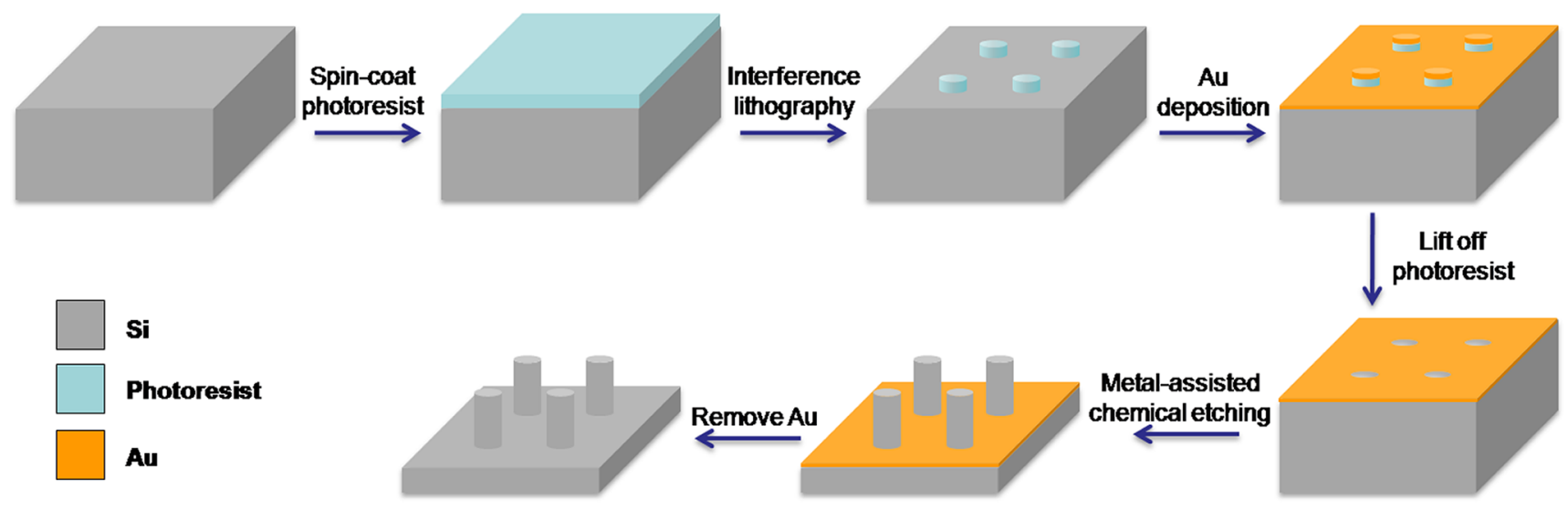

(a)
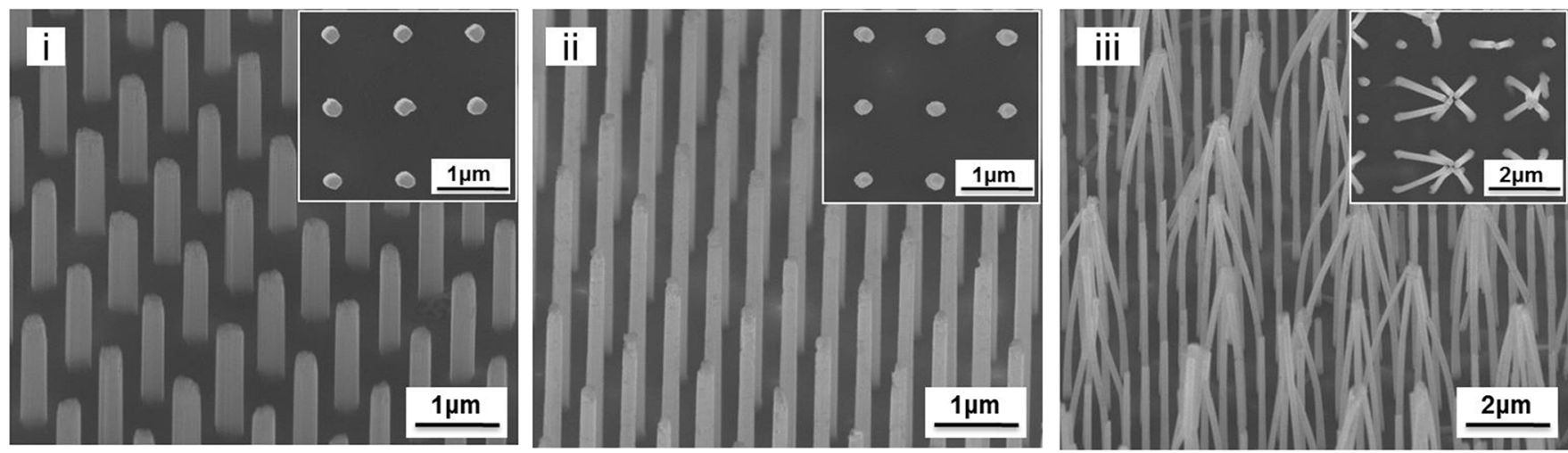

(b)

Figure 1. (a) Schematic diagram of the process flow for fabrication of Si nanopillars using the IL-MACE method. (b) SEM images of Si nanopillars with heights of (i) $\sim 2 \mu \mathrm{m}$, (ii) $\sim 4 \mu \mathrm{m}$, and (iii) $\sim 7 \mu \mathrm{m}$, respectively. The insets are top-view SEM images of the respective samples. All samples in part $\mathrm{b}$ have the same period of $1 \mu \mathrm{m}$.

deviated from theory. A comprehensive review on wicking can be found in ref 22 .

Recently, we have discovered a simple method of creating ordered arrays of silicon nanopillars over large areas $\left(1 \mathrm{~cm}^{2}\right)$ by means of interference lithography and metal-assisted chemical etching (IL-MACE) method. ${ }^{23,24}$ The height of the nanopillars can be easily varied by controlling the etching time. The objective of the present work is to examine the effect of nanopillar geometry on the dynamics of wicking theoretically and to validate the theoretical predictions with experimental results obtained from our samples prepared by the IL-MACE method. We have also extended the comparison of theoretical predictions to data published in the literature.

\section{EXPERIMENTAL DETAILS}

The process flow for the synthesis of $\mathrm{Si}$ nanopillars using the ILMACE technique is illustrated in Figure 1a. P-type (100) Si wafers were first cleaned by standard RCA1 and RCA 2 processes and dipped in $10 \% \mathrm{HF}$ for $1 \mathrm{~min}$ to remove native oxide. The Si wafers were then coated with photoresist (Ultra-i 123) approximately $300 \mathrm{~nm}$ thick, and cured at $90{ }^{\circ} \mathrm{C}$ for $90 \mathrm{~s}$. The photoresist was exposed using a Lloyd'smirror-type interference lithography setup with a $\mathrm{HeCd}$ laser source $(\lambda$ $=325 \mathrm{~nm}){ }^{25}$ Two perpendicular exposures of $60 \mathrm{~s}$ duration were then performed on the samples, which was followed by a postbake at 110 ${ }^{\circ} \mathrm{C}$ for $60 \mathrm{~s}$. The exposed photoresist was removed using Microposit
MF CD-26 developer and circular-shape photoresist dots were formed on the $\mathrm{Si}$ surface. The samples were then subjected to an oxygen plasma etch (power of $200 \mathrm{~W}$, oxygen pressure of $0.2 \mathrm{mbar}$, etching time of $30-45 \mathrm{~s}$ ) to remove the residual photoresist on the Si surface, and to reduce the size of the photoresist dots. Au was thermally evaporated on the substrate to a thickness of $\sim 30 \mathrm{~nm}$, at a pressure of $10^{-6}$ Torr. The photoresist dots were lifted off by ultrasonic bath in acetone for 15-20 min, leaving an $\mathrm{Au}$ mesh on the silicon surface. The samples were etched in a solution of $\mathrm{H}_{2} \mathrm{O}, \mathrm{HF}$, and $\mathrm{H}_{2} \mathrm{O}_{2}$ at room temperature for 5-30 min. The concentrations of $\mathrm{HF}$ and $\mathrm{H}_{2} \mathrm{O}_{2}$ were 4.6 and $0.44 \mathrm{M}$, respectively. The samples were then immersed in a standard $\mathrm{Au}$ etchant for $5 \mathrm{~min}$ to remove $\mathrm{Au}$ and dried by a nitrogen gun after rinsing in deionized water.

Figure $1 \mathrm{~b}$ shows the scanning electron microscope (SEM) images of silicon nanopillars obtained with an FEI NOVA SEM230 instrument. All samples were fabricated with exactly the same conditions except for the duration of etching $(5-30 \mathrm{~min})$, which gave the nanopillars different heights on different samples. Note that nanopillars from samples etched for 5-20 min are perfectly straight (see Figure $1 \mathrm{~b}$, i and ii). However, when the nanopillars become too tall $(\sim 7 \mu \mathrm{m})$, the high aspect ratio causes the mechanical strength of the nanopillars to be reduced significantly so that capillary forces can now overcome the elastic forces and cause sufficient permanent deformation/clumping of the nanopillars (see Figure 1b, iii). ${ }^{26}$

To examine the wicking characteristics, the samples were placed vertically, and a droplet $(1 \mu \mathrm{L})$ of deionized water or silicone oil (HIVAC F4) was placed at the bottom of the sample surface. The 
wicking process was recorded with a high speed camera (Photron Fastcam SA5) operating at 1000 frames per second and analyzed by means of software (Photron FASTCAM Viewer).

\section{MODEL}

Bico et al. ${ }^{13}$ previously established the condition for wicking to take place as

$$
\cos \theta>\cos \theta_{\mathrm{c}}=\frac{1-\phi_{\mathrm{s}}}{r-\phi_{\mathrm{s}}}
$$

where $\theta$ is the contact angle the liquid makes with a flat surface of the substrate material, $\theta_{c}$ is the critical angle $\left(0^{\circ} \leq \theta_{c} \leq 90^{\circ}\right)$, $r$ is the roughness of the textured surface (ratio of the actual surface area to projected area) and $\phi_{s}$ is the ratio of the area of the top of the nanopillars (which was assumed to remain dry) to the projected area.

In the case of our nanopillars,

$$
\begin{aligned}
& r=\frac{\pi d h}{(d+s)^{2}}+1 \\
& \phi_{s}=\frac{\pi d^{2}}{4(d+s)^{2}}
\end{aligned}
$$

where $d$ is the diameter and $h$ is the height of the nanopillars, and $s$ is the spacing between nanopillars (i.e., the period of the nanopillars is $d+s$ ).

The displacement of the wicking front with time was found to follow the well-known diffusive relation

$$
z=(D t)^{1 / 2}
$$

where $z$ is the displacement of the wicking front, $D$ is a coefficient independent of $z$ and $t$, and $t$ refers to the time after the start of wicking. Bico et al. derived $D$ by assuming Poiseuille flow and equating the driving capillary pressure for wicking with the retarding pressure associated with viscous losses, obtaining

$$
D=\frac{2 \gamma h}{3 \mu \beta} \frac{\cos \theta-\cos \theta_{c}}{\cos \theta_{c}}
$$

where $\gamma$ is the surface tension of the liquid, $\mu$ is the viscosity of the liquid, and $\beta$ is an empirically determined constant that is multiplied with the viscous loss pressure derived from Poseuille flow of a fluid of height $h$ over a flat plane. ${ }^{13}$ The purpose of this multiplication is to account for the enhancement of viscous losses due to the presence of micropillars on the plane.

From eq 5 , it can be seen that aside from topographical $(h, \beta$, $\left.\theta_{c}\right)$ and chemical parameters $(\theta)$ of the surface, the properties of the liquid $(\gamma, \mu)$ also play a role in determining the wicking velocity. Because the driving pressure for wicking is proportional to $\gamma$ and the viscous pressure retarding wicking is proportional to $\mu$, wicking velocity is dependent on $\gamma / \mu$. This dependence can be intuitively verified by considering the two extreme cases, $\gamma \gg \mu$ (e.g., water) and $\gamma \ll \mu$ (e.g., polydimethylsiloxane (PDMS)), which will result in very high and very low velocities, respectively.

However, unlike values of $\gamma$ and $\mu$, which are readily available for a wide range of liquids, $\beta$ has to be experimentally determined and as a result, $D$ in eq 5 cannot be fully predicted with theory alone. Thus, it is difficult to gain a full insight of how geometrical parameters of the nanopillars affect $D$, which in turn determines the wicking kinematics. In this paper, we attempt to derive an analytical solution for $\beta$ and verify that with experimental data.

To simplify the derivation, we start by approximating the flow of fluid through the nanopillars as flow through open nanochannels that are of the same height, $h$, and length, $(d+s)$, as a unit cell of nanopillar as shown in Figure 2. A unit cell of
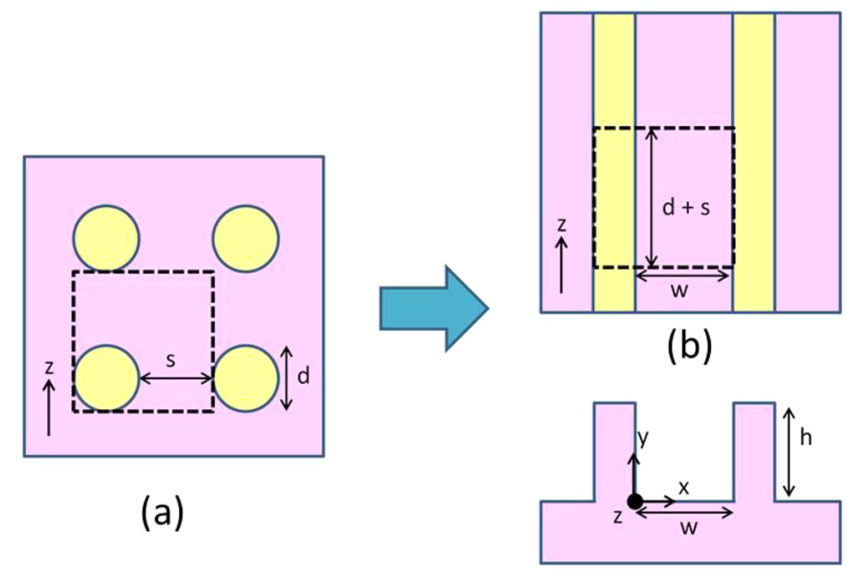

(c)

Figure 2. Approximating a unit cell (indicated by dashed black lines) of nanopillars as a unit cell of nanochannel that holds the same volume of liquid. (a) Top view of a unit cell; $(b, c)$ top view and side view of a nanochannel. The yellow regions indicate the top of the nanostructures at $y=h$, which remain dry throughout the wicking process, while the violet regions indicate the bottom regions at $y=0$. Flow of fluid is in the $z$-direction in all cases.

nanochannel can hold the same volume of fluid as a unit cell of nanopillar and, thus, the width of the nanochannel, $w$, can be calculated to be

$$
w=\frac{\left[(d+s)^{2}-\frac{\pi}{4} d^{2}\right] h}{(d+s) h}=\frac{(4-\pi) d^{2}+8 d s+4 s^{2}}{4(d+s)}
$$

For steady state, incompressible flow in the $z$-direction through an open channel such as that shown in Figure $2 \mathrm{c}$ and ignoring gravitational effects, which are relatively insignificant at the scale of our experiments, ${ }^{13}$ the Navier-Stokes' equation can be reduced to

$$
-\frac{\Delta P}{\mu z}=\left(\frac{\partial^{2} U}{\partial x^{2}}+\frac{\partial^{2} U}{\partial y^{2}}\right)
$$

where $\Delta P$ is the capillary pressure driving the flow and $U(x, y)$ is the velocity profile. Following Bico et al.'s derivation, $(\Delta P) / z$ is independent of $x$ and $y$ and, thus, when eq 7 is solved with the boundary conditions

$$
\begin{aligned}
& U(0, y)=0 \\
& U(x, 0)=0 \\
& U(w, y)=0 \\
& U(x, 2 h)=0
\end{aligned}
$$

the velocity profile can be given as 


$$
\begin{aligned}
& U(x, y)= \\
& \sum_{m, n=0}^{\infty} \frac{16 \Delta P}{\left[\frac{(2 m+1)^{2}}{w^{2}}+\frac{(2 n+1)^{2}}{4 h^{2}}\right](2 m+1)(2 n+1) \pi^{4} \mu z} \\
& \quad \sin \frac{(2 m+1) \pi x}{w} \sin \frac{(2 n+1) \pi y}{2 h}
\end{aligned}
$$

Details of the derivation of eq 9 can be found in SI.1 of the Supporting Information. Averaging $U(x, y)$ over the area of $(w$ $\times h)$ to find the mean velocity $U_{\text {mean }}$, we obtain

$$
\begin{aligned}
U_{\text {mean }} & =\frac{\int_{0}^{h} \int_{0}^{w} U(x, y) \mathrm{d} x \mathrm{~d} y}{w h} \\
= & \sum_{m, n=0}^{\infty} \frac{64 \Delta P}{\left[\frac{(2 m+1)^{2}}{w^{2}}+\frac{(2 n+1)^{2}}{4 h^{2}}\right](2 m+1)^{2}(2 n+1)^{2} \pi^{6} \mu z}
\end{aligned}
$$

Since the value of each term in the summation series in eq 10 decreases exponentially with increasing $m$ or $n$, we can approximate $U_{\text {mean }}$ by considering only the first term $(m=0$, $n=0)$ of the summation series which contributes the most to the value of $U_{\text {mean }}$. We have carried out a systematic analysis of the effect on the contribution of other terms (where $m>1$ and $n>1$ ) in eq 10 and found that our assumption to only consider the first term in the summation series is justified. Details of this analysis can be found in SI.2 of the Supporting Information.

To take into account the value added to $U_{\text {mean }}$ by the rest of the terms, we multiply by a constant $C$ to approximate the value of $U_{\text {mean }}$. Thus, we have

$$
U_{\text {mean }} \approx C \frac{64 \Delta P}{\left[\frac{1}{w^{2}}+\frac{1}{4 h^{2}}\right] \pi^{6} \mu z}
$$

To find $C$, we consider the extreme case where $w$ approaches infinity. In this instance, the flow of fluid is equivalent to flow on a flat plane, and, thus, eq 11 will be reduced to Poiseuille flow over a flat plane, which is given by ${ }^{13}$

$$
U_{\text {mean }}=\frac{\Delta P h^{2}}{3 \mu z}
$$

Equating eq 11 when $w \rightarrow \infty$ and eq 12 , we find that $C=\pi^{6}$ / 768. Substituting this into eq 11 we obtain

$$
U_{\text {mean }} \approx \frac{\Delta P h^{2} w^{2}}{3 \mu z\left[w^{2}+4 h^{2}\right]}
$$

Finally, by comparing eq 13 with the mean velocity derived by Bico et al. $\left(U_{\text {mean }}=\left(\Delta P h^{2}\right) /(3 \beta \mu z)\right),{ }^{13}$ we arrive at an expression for $\beta$ as

$$
\beta=\frac{4 h^{2}}{w^{2}}+1
$$

From eqs 6 and 14, it can be seen that when the surface approaches a flat topography $(s \gg d$ and $s \gg h ; h \ll d$ and $h \ll$ $s), \beta$ approaches 1 , i.e., the flow approaches Poiseuille's flow on a flat plane. On the other hand, when the surface approaches a very rough texture ( $h \gg d$ and $h \gg s$ ), $\beta$ approaches infinity, which is to be expected, taking into consideration the enormous viscous losses associated with very tall or very dense arrays of pillars. Note that this does not necessarily mean that wicking speed will be very low, as the geometric parameters also influence the driving pressure for wicking. This will be discussed in more details later in the paper. For now, we consider another case, $d \gg s$, for which $\beta$ will become inversely related to $d$. This can be explained by considering the interstitial spaces formed when a rectangular array of cylindrical pillars join up; as the size of these pillars increase, the interstitial spaces for wicking to take place become more spacious and deterrence to wicking falls. In the extreme case where $d \gg h, \beta$ approaches 1 as viscous losses to the sidewalls of the pillars become negligible and the flow returns to one on a flat plane. Extending eq 6 to square pillars so that $w=(d s /(d+s))+s$, it is easy to see that for the same case of $d \gg s, \beta$ is now inversely related to $s$ instead. This is not surprising because unlike cylindrical pillars, there are no interstitial spaces when square pillars join up. As a result, the flow becomes more constricted (higher $\beta$ ) as $s$ decreases, and in the extreme case where $s=0, \beta$ approaches infinity, which is consistent with the expectation that wicking cannot occur on a flat surface. ${ }^{13}$ The above considerations show that eq 14 supports common, intuitive knowledge of wicking for limiting cases, thereby providing a preliminary verification of the validity and versatility of eq 14 . We will revisit eq 14 later in this paper when we compare the theoretical to experimental values of $\beta$.

\section{RESULTS AND DISCUSSION}

On the basis of eqs 1-3, we have calculated the critical contact angles for our samples, and the values are listed in Table 1 .

Table 1. Critical Contact Angle $\left(\boldsymbol{\theta}_{\mathrm{c}}\right)$ for Silicon Nanopillars Fabricated by the IL-MACE Method

\begin{tabular}{lcccccc} 
& $\begin{array}{c}\text { etch time } \\
(\mathrm{mins})\end{array}$ & $\begin{array}{c}\text { diameter } \\
(\mu \mathrm{m})\end{array}$ & $\begin{array}{c}\text { height } \\
(\mu \mathrm{m})\end{array}$ & $\phi_{\mathrm{s}}$ & $r$ & $\theta_{\mathrm{c}}\left(^{o}\right)$ \\
pillar A & 5 & 0.275 & 0.75 & 0.043 & 1.897 & 59.62 \\
pillar B & 10 & 0.300 & 2.00 & 0.064 & 2.710 & 69.28 \\
pillar C & 20 & 0.300 & 4.20 & 0.064 & 4.590 & 78.07 \\
pillar D & 30 & 0.300 & 7.00 & 0.064 & 6.984 & 82.23 \\
\hline
\end{tabular}

Having found that the contact angles for water and silicone oil on flat silicon substrate are $\theta_{\text {water }}=56.3^{\circ}$ and $\theta_{\text {oil }}=18.0^{\circ}$, respectively, we are able to verify that Bico et al.'s condition for wicking $\left(\theta_{c}>\theta\right)$ agrees with our experiments. Water and silicone oil were able to wick in all the samples except sample A (for water only), which had a critical angle that is fairly close to the contact angle of water on a flat silicon surface. Since the driving pressure for wicking ${ }^{13}$ is proportional to $(\cos \theta-\cos$ $\left.\theta_{c}\right) / \cos \theta_{\mathcal{c}}$ it is not surprising to find that wicking may not occur at all when $\cos \theta \approx \cos \theta_{\mathcal{c}}$, especially in the presence of slight surface defects or impurities, which tend to raise the energy cost of wicking.

Next, we study the wetting properties of the silicon nanopillars surfaces by examining the wicking dynamics of silicone oil $\left(\gamma=3.399 \times 10^{-2} \mathrm{~N} / \mathrm{m}, \mu=3.94 \times 10^{-2}\right.$ Pas, $\theta_{\text {oil }}=$ $\left.18^{\circ}\right)^{27}$ and water $\left(\gamma=7.28 \times 10^{-2} \mathrm{~N} / \mathrm{m}, \mu=8.9 \times 10^{-3} \mathrm{Pas}\right.$, $\left.\theta_{\text {water }}=56.32^{\circ}\right)$. Figure 3 shows photographs of the spreading of $1 \mu \mathrm{L}$ oil droplet pipetted onto an upright silicon substrate with nanopillar texture on its surface. The liquid reached the top of the sample (which is about $8 \mathrm{~mm}$ in height) in about 300 s. For water, the wicking was much faster, and it reached the end of the sample in about 10s. The videos of the wicking of water and silicone oil are provided in the Supporting Information as "Video-Water" and "Video-Oil", respectively. 


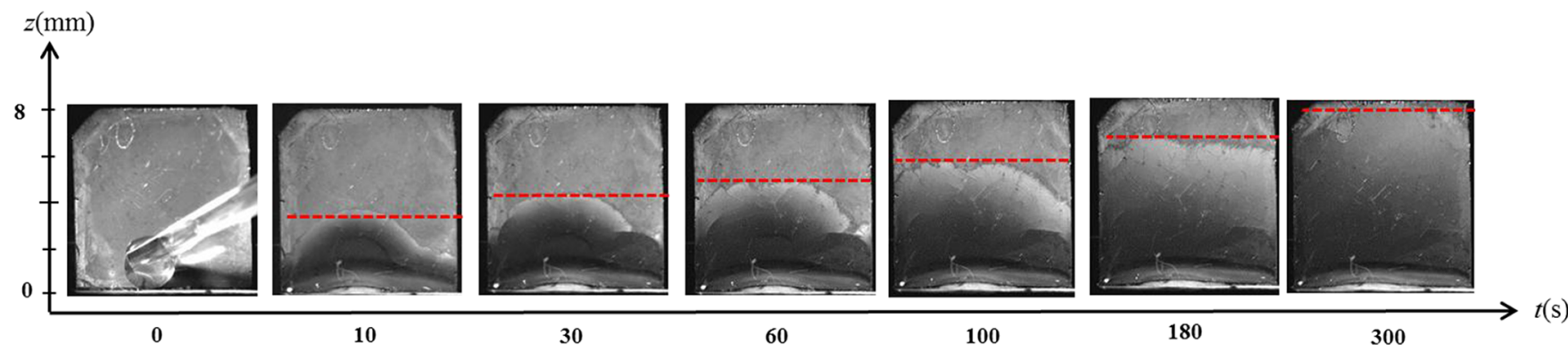

Figure 3. Snapshots of the wicking process of silicone oil on silicon nanopillar surface (sample B). The red dotted line marks the liquid front.

The difference in the wicking speed can be accounted for by the difference in $\gamma / \mu$ (see eq 5 ) of the two liquids.

Figure 4 shows plots of distance traveled by the wetting front versus the square root of time (i.e., $z$ vs $t^{1 / 2}$ ) for all our samples.

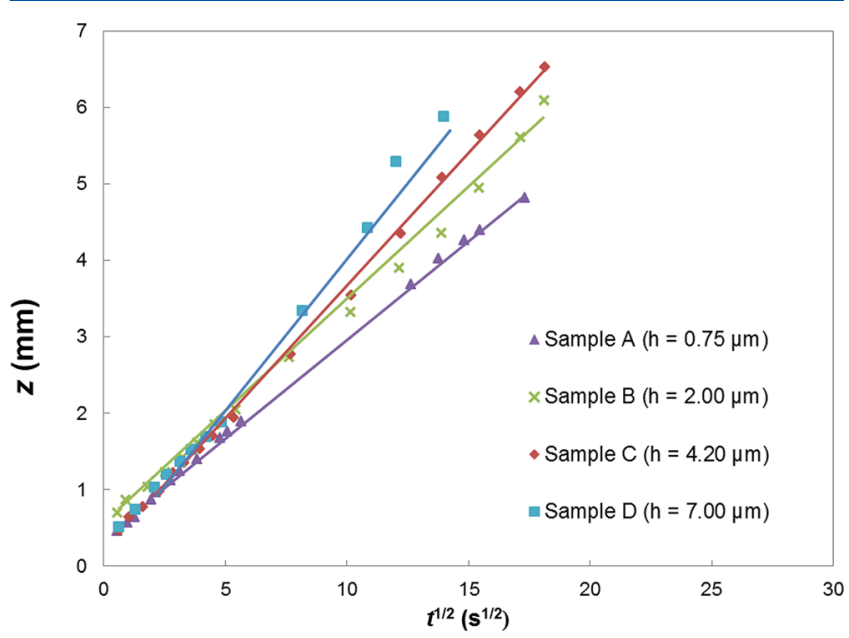

Figure 4. Plot of distance traveled by the wetting front against the square root of time for nanopillars wetted with silicone oil $(\gamma=3.399$ $\times 10^{-2} \mathrm{~N} / \mathrm{m}, \mu=3.94 \times 10^{-2}$ Pas, $\left.\theta_{\text {oil }}=18^{\circ}\right)$.

As with previous studies, ${ }^{13,18,19}$ the linear trendlines do not continue through the point of origin. This deviation in the early stages of wicking may be due to the influence of other processes that are observed to take place right before or during the onset of wicking (e.g., droplet spreading). Note that the viscous enhancement factor $\beta$ can be obtained by measuring the gradient of this plot (i.e., the value of $D^{1 / 2}$ ) and substituting the value into eq 5 .

The calculated (from eq 14) and experimentally determined values of $\beta$ are plotted in Figure 5. As can be seen from this figure, our theoretical values for $\beta$ agree very well with the experimental data over a wide spectrum of $h / w$ values. Note also that the empirical values of $\beta$ obtained with water and silicone oil are very close, thus proving that $\beta$ is independent of fluid properties, as predicted by eq 14 . The only exception to this is the data point at $h / w=7.1$ where $\beta$ (water) deviates from $\beta$ (silicone oil). This difference is likely due to the deformations of the mechanically weak, high aspect ratio $(\approx 23)$ nanopillars caused by capillary forces during the wicking process (see Figure 1b, iii).

In Figure 5, we also show the $\beta$ values calculated for our samples based on the models proposed by Ishino et al. ${ }^{14}$ and Zhang et al. ${ }^{1}$ We have taken $U_{\text {mean }}$ reported in these papers and compared with Bico's expression $\left(U_{\text {mean }}=\left(\Delta P h^{2}\right) /(3 \beta \mu z)\right)$ to obtain $\beta$. This will give

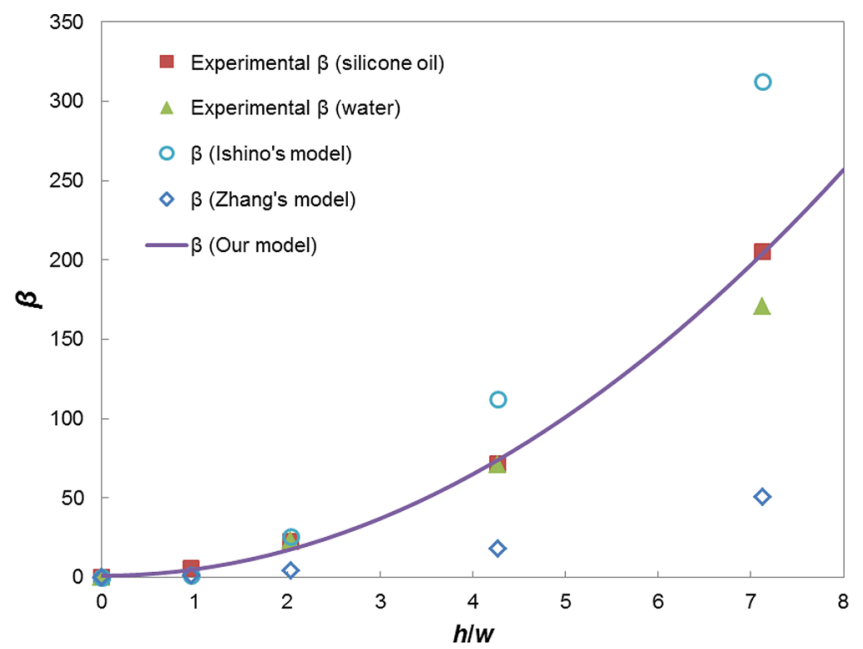

Figure 5. Experimental and calculated values of $\beta$. Data points for $\beta$ (silicone oil) and $\beta$ (water) are obtained with silicone oil and water, respectively. Calculation based on our method is represented by a solid line. Also shown in this figure are the calculated $\beta$ values of our samples based on the models of Zhang et al. ${ }^{1}$ and Ishino et al. ${ }^{14}$

$$
\begin{aligned}
& \beta=\frac{1}{1-\phi_{s}} \text { for } h<(d+s) \\
& \beta=\frac{4 \pi h^{2}}{3\left(1-\phi_{s}\right)(d+s)^{2}\left[\ln \left(\frac{2 d+2 s}{d}\right)-1.31\right]} \text { for } \\
& \quad h>(d+s)
\end{aligned}
$$

for Ishino et al.'s ${ }^{14}$ derivation, and

$$
\beta=\frac{4 d h^{2}}{(d+s)^{2}}
$$

for Zhang et al.'s ${ }^{1}$ derivation.

It can be observed that Zhang et al.'s approximation for $\beta$ underestimates its true value for all $h / w$, while Ishino et al.'s expressions overestimate the experimental $\beta$ at high $h / w(\geq 4)$ but underestimate it at low $h / w(<2)$. The reason for the deviations can be attributed to the models that were considered. For instance, Zhang et al. estimated the flow between the pillars to be Poiseuille's flow between two parallel plates (the pillars being the plates) and ignored the contribution to the viscous losses by the floor at $y=0$. In addition, viscous losses were only considered to occur when the flow occurs between two pillars. However, the velocity profile when the flow proceeds past the channels between adjacent pillars is not expected to be spatially constant, and thus there 
should also be shear losses involved in those regions. For these reasons, the $\beta$ values determined from Zhang et al.'s model are lower than the experimental values. As for Ishino et al.'s method, when $h<(d+s)$, the flow between micropillars is assumed to be similar to flow over a flat plane. In this case, it is expected that the calculated $\beta$ values should be smaller than the experimental values because the effect of micropillars on viscous losses was not considered. For $h>(d+s)$, Ishino et al. suggested that the micropillars can be considered to be infinite, and thus the calculated $\beta$ values are anticipated to be larger than the actual values.

To further validate our theory, we calculate the $\beta$ values for the micropillars reported by Ishino et al. ${ }^{14}$ The theoretical predictions by Ishino et al. and Zhang et al. are presented alongside our calculated $\beta$ values and the experimental data for comparison purposes. The results are plotted in Figure 6. It can

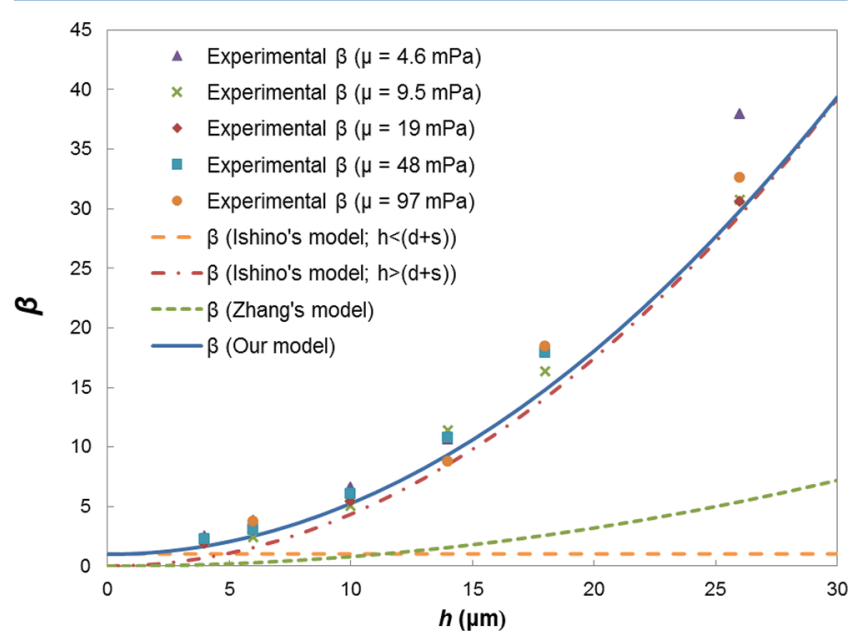

Figure 6. Comparison of $\beta$ values obtained by our method and other models for the experimental data presented in Ishino et al.'s paper. Experimental and theoretical values are plotted as points and lines, respectively. Our model is represented by the solid blue line. Five different test liquids $\left(\gamma=2 \times 10^{-2} \mathrm{~N} / \mathrm{m}\right)$ were used, and their respective viscosities are given in the legend. $d=2 \mu \mathrm{m}$ and $s=8 \mu \mathrm{m}$ remained constant for all experiments.

be seen that our model fits Ishino et al.'s experimental data very well, and shows a significant improvement over Ishino et al.'s theoretical estimation of $\beta$ values when $h$ is small. Interestingly, our theory (eq 14) and Ishino et al.'s model (eq 15.2) converge when $h$ increases. However, this is only coincidental as it can be proven mathematically that this is not true for all $d$ and $s$ values. In addition, Ishino et al.'s results agree with our conclusion that $\beta$ is independent of the properties of the test fluid.

Our model (eq 14) can also be employed to solve an observation previously made by Ishino et al., namely, the diffusion coefficient $D$ becomes increasingly independent of nanopillar height, $h$, as $h$ becomes very large. By considering $h$ $\gg d, s, w$ in eqs $1-3$ and 14 , it can be shown that $(\cos \theta-\cos$ $\left.\theta_{c}\right) / \cos \theta_{c} \sim h$ and $\beta \sim h^{2}$. Therefore, from eq 5, D will approach a constant value. This means that when the micro/ nanopillars are short, the capillary driving force for wicking is increased more than viscous losses (represented by $\beta$ ) with increasing pillar height, thus resulting in faster wicking velocity. However, as the pillars become taller, the viscous force is increasing as fast as the capillary driving force with increasing height, and therefore a maximum speed will be reached. The understanding that wicking velocity cannot be endlessly improved by increasing $h$ has important implications for the designing of engineering applications based on wicking and is one of the many insights that can be derived from this model.

\section{CONCLUSIONS}

We presented in this paper a theoretical study and an experimental validation of the wicking dynamics on a square array of silicon nanopillars. The enhancement factor of viscous losses, $\beta$, due to the presence of nanopillars was investigated and was found to depend on the ratio of $h / w$ where $w$ is the width of the channel that is used to approximate the wicking flow through nanostructures. Our expression for $\beta$ was also found to be applicable to published results obtained by other groups.

The success in describing the dynamics of wicking explicitly without any empirical parameters means that the wicking property (i.e., speed) of a particular material can be adjusted by simply controlling the geometry (e.g., height) of the textured surface. However, there is a limit when using the height of micro/nanostructures to do so, as the wicking speed will approach an asymptotic value when the height of the structures becomes much greater than the other dimensions. These insights are most valuable for the designing of future wicking applications.

\section{ASSOCIATED CONTENT}

\section{Supporting Information}

The Supporting Information provides derivations of the equations in the text as well as additional analysis. Videos in AVI format showing the wicking of silicone oil and water are available. This information is available free of charge via the Internet at http://pubs.acs.org.

\section{AUTHOR INFORMATION}

\section{Corresponding Author}

*E-mail: elechoi@nus.edu.sg.

\section{Author Contributions}

The manuscript was written through contributions of all authors.

Notes

The authors declare no competing financial interest.

\section{ACKNOWLEDGMENTS}

The authors would like to acknowledge the partial funding of this work by the Singapore-MIT Alliance. T.T.M., C.Q.L., and H.Z. would like to express their deepest gratitude to the National University of Singapore, Singapore-MIT Alliance, and GLOBALFOUNDRIES for provision of research scholarships.

\section{REFERENCES}

(1) Zhang, C.; Hidrovo, C. H. Investigation of Nanopillar Wicking Capabilities for Heat Pipes Applications. ASME 2009 Second Int. Conf. Micro/Nanoscale Heat Mass Transfer 2009, 3, 423-437.

(2) Ding, C.; Bozorgi, P.; Meinhart, C. D.; MacDonald, N. C. Wicking Optimization for Thermal Cooling. Hiltonhead Conference 2010, Solid-State Sens., Actuators, Microsyst. Workshop 2010, 376.

(3) Oshman, C.; Li, Q.; Liew, L.-A.; Yang, R.; Lee, Y. C.; Bright, V. M.; Sharar, D. J.; Jankowski, N. R.; Morgan, B. C. Thermal Performance of a Flat Polymer Heat Pipe Heat Spreader Under High Acceleration. J Micromech. Microeng. 2012, 22, 045018.

(4) Kang, S.-W.; Tsai, S.-H.; Ko, M.-H. Metallic Micro Heat Pipe Heat Spreader Fabrication. Appl. Therm. Eng. 2004, 24, 299-309. 
(5) Pararas, E. E. L.; Borkholder, D. A.; Borenstein, J. T. Microsystems Technologies for Drug Delivery to the Inner Ear. Adv. Drug Delivery Rev. 2012, DOI: 10.1016/j.addr.2012.02.004.

(6) Guillaume, D. W.; DeVries, D. Improving the Pneumatic Nebulizer by Shaping the Discharge of the Capillary Wick. J. Biomed. Eng. 1991, 13, 526-528.

(7) Liu, C.; Mauk, M. G.; Hart, R.; Qiu, X.; Bau, H. H. A Self-Heating Cartridge for Molecular Diagnostics. Lab Chip 2011, 11, 2686-2692.

(8) Araŭjo, A. C.; Song, Y.; Lundeberg, J.; Ståhl, P. L.; Brumer, H. Activated Paper Surfaces for the Rapid Hybridization of DNA through

Capillary Transport. Anal. Chem. 2012, 84, 3311-3317.

(9) Lankelma, J.; Nie, Z.; Carrilho, E.; Whitesides, G. M. Paper-Based Analytical Device for Electrochemical Flow-Injection Analysis of Glucose in Urine. Anal. Chem. 2012, 84, 4147-4152.

(10) Lazarus, N.; Bedair, S. S.; Lo, C.-C.; Fedder, G. K. CMOSMEMS Capacitive Humidity Sensor. J. Microelectromech. Syst. 2010, 19, 183-191.

(11) Peng, P.; Summers, L.; Rodriguez, A.; Garnier, G. Colloids Engineering and Filtration to Enhance the Sensitivity of Paper-Based Biosensors. Colloids Surf. B 2011, 88, 271-278.

(12) Feng, X. J.; Jiang, L. Design and Creation of Superwetting/ Antiwetting Surfaces. Adv. Mater. 2006, 18, 3063-3078.

(13) Bico, J.; Tordeux, C.; Quéré, D. Rough Wetting. Europhys. Lett. 2001, 55, 214-220.

(14) Ishino, C.; Reyssat, M.; Reyssat, E.; Okumura, K.; Quéré, D. Wicking within Forests of Micropillars. Europhys. Lett. 2007, 79, 56005 .

(15) Martines, E.; Seunarine, K.; Morgan, H.; Gadegaard, N.; Wilkinson, C. D. W.; Riehle, M. O. Superhydrophobicity and Superhydrophilicity of Regular Nanopatterns. Nano Lett. 2005, 5, 2097-2103.

(16) Extrand, C. W.; Moon, S. I.; Hall, P.; Schmidt, D. Superwetting of Structured Surfaces. Langmuir 2007, 23, 8882-8890.

(17) Ahn, H. S.; Park, G.; Kim, J.; Kim, M. H. Wicking and Spreading of Water Droplets on Nanotubes. Langmuir 2012, 28, 2614-2619.

(18) Vorobyev, A. Y.; Chunlei, G. Laser Turns Silicon Superwicking. Opt. Exp. 2010, 18, 6455-6460.

(19) Vorobyev, A. Y.; Chunlei, G. Water Sprints Uphill on Glass. J. Appl. Phys. 2010, 108, 123512.

(20) Washburn, E. W. The Dynamics of Capillary Flow. Phys. Rev. 1921, 17, 273-283.

(21) Hay, K. M.; Dragila, M. I.; Liburdy, J. Theoretical Model for the Wetting of a Rough Surface. J. Colloid Interface Sci. 2008, 325, 472477.

(22) Quéré, D. Wetting and Roughness. Annu. Rev. Mater. Res. 2008, $38,71-99$.

(23) Choi, W. K.; Liew, T. H.; Dawood, M. K.; Smith, H. I.; Thompson, C. V.; Hong, M. H. Synthesis of Silicon Nanowires and Nanofin Arrays Using Interference Lithography and Catalytic Etching. Nano Lett. 2008, 8, 3799-3802.

(24) Dawood, M. K.; Liew, T. H.; Lianto, P.; Hong, M. H.; Tripathy, S.; Thong, J. T. L.; Choi, W. K. Interference Lithographically Defined and Catalytically Etched, Large-Area Silicon Nanocones from Nanowires. Nanotechnology 2010, 21, 205305.

(25) Savas, T. A.; Schattenburg, M. L.; Carter, J. M.; Smith, H. I. Large-Area Achromatic Interferometric Lithography for $100 \mathrm{~nm}$ Period Gratings and Grids. J. Vac. Sci. Technol. 1996, B14, 4167-4170. (26) Dawood, M. K.; Zheng, H; Kurniawan, N. A.; Leong, K. C.; Foo, Y. L.; Rajagopalan, R.; Khan, S. A.; Choi, W. K. Modulation of Surface Wettability of Superhydrophobic Substrates using Si Nanowire Arrays and Capillary-Force-Induced Nanocohesion. Soft Matter 2012, 8, 3549-3557.

(27) Data sheet for silicone oil. http://www.silicone.jp/e/products/ type/oil/index.shtml. 